\title{
A!
}

This is an electronic reprint of the original article.

This reprint may differ from the original in pagination and typographic detail.

Gallo, Pasquale

\section{Some Considerations on Stress Intensity Factor at Atomic Scale}

Published in:

ICTAEM 2020 : Proceedings of the Third International Conference on Theoretical, Applied and Experimental Mechanics

DOI:

10.1007/978-3-030-47883-4_57

Published: 01/01/2020

Document Version

Peer reviewed version

Published under the following license:

Unspecified

Please cite the original version:

Gallo, P. (2020). Some Considerations on Stress Intensity Factor at Atomic Scale. In ICTAEM 2020 :

Proceedings of the Third International Conference on Theoretical, Applied and Experimental Mechanics (pp. 319-324). (Structural Integrity; Vol. 16). SPRINGER. https://doi.org/10.1007/978-3-030-47883-4_57

This material is protected by copyright and other intellectual property rights, and duplication or sale of all or part of any of the repository collections is not permitted, except that material may be duplicated by you for your research use or educational purposes in electronic or print form. You must obtain permission for any other use. Electronic or print copies may not be offered, whether for sale or otherwise to anyone who is not an authorised user. 


\title{
Some Considerations on Stress Intensity Factor at Atomic Scale
}

\author{
Pasquale Gallo 1 [0000-0001-5742-8647] \\ ${ }^{1}$ Aalto University, P.O. Box 14100, FI-00076 AALTO, Finland \\ pasquale.gallo@aalto.fi
}

\begin{abstract}
This work reviews recent molecular statistics (MS) numerical experiments of cracked samples, and discusses the crack-tip region stress field of ideal brittle materials. Continuum-based linear elastic fracture mechanics, indeed, breaks down at extremely small scale, where the discrete nature of atoms is considered. Surprisingly, recent results have shown that the concept of stress intensity factor (SIF) is still valid. In this work, by means of MS simulations on single-edge cracked samples of ideal brittle silicon, it is shown that the stress intensity factor derived from the virial stress may be useful to describe the fracture at extremely small dimensions and to quantify the breakdown of continuum-based linear elastic fracture mechanics. However, it is still debated whether a continuum-based concept such as the "stress" should be applied to a system made of atoms.
\end{abstract}

Keywords: Virial Stress, Atom, Crack, Stress Intensity Factor.

\section{Introduction}

With recent developments in miniaturization of electronics devices such as nano and micro-electromechanical systems (NEMS/MEMS), issues usually addressed at the macroscale, i.e. fatigue and fracture, have been brought into a completely new "scale" $[1,2]$. At the same time, developments in nanotechnology give nowadays a completely new way at which the fatigue and fracture can be studied with a remarkable potential impact in several fields of engineering [3-10]. At such small scales, where discrete nature of atoms can't be ignored, the continuum concepts largely used at the macroscale become questionable [11-13].

While methods based on energy have shown good potential to be extended from continuum to discrete system $[12,14-18]$, it is still debated whether the concept of "stress" should be applied to a system made of atoms [19, 20]. Stress is, indeed, a continuum concept, originated from the study of strength and failure of solid, and commonly defined as the quantity that represents the internal forces on a defined plane of a continuous material. Thus, questions arise on the definition of "atomic stress". When investigating fracture at the atomic scale, the virial stress tensor is commonly employed to derive mechanical stresses acting on atoms [20-22]. Setting aside the debate on the validity of the virial stress as a representation of mechanical 
stress at atomic scale, in the present work the focus is on the applicability of classic continuum concepts in the presence of defects, such as the stress intensity factor (SIF). By reviewing recent molecular statistics (MS) analyses on single-edged cracked samples loaded under mode I [23], and representative of ideal brittle fracture, it is demonstrated that the virial stress shows the trend of inverse square root singularity and that computation of the SIF according to Irwin's concept is possible. Furthermore, the breakdown of continuum linear elastic fracture mechanics, recently defined by means of energy concepts [11-13], is here quantified by using merely the stress fields.

It is concluded that the SIF from atomic stress may be useful in characterizing fracture at atomic scale, provided that the virial stress is accepted as representative of atomic mechanical stress. On the other hand, energy concepts should be anyway preferred for future developments, since they can provide a direct equivalence between continuum, discrete systems and among different scales.

\section{Review of Recent Molecular Statistics Simulations on Cracked Samples}

Recently, fracture tests by means of MS simulations were conducted by using opensource code LAMMPS [24]. While details can be found in [23], important aspects are presented hereafter. The modified Stillinger-Weber (SW) interatomic potential [25] was employed. The SW potential is representative of ideal brittle fracture, and often used when studying single crystal silicon. The focus was on single-edge cracked sample, loaded under mode I. Figure 1 depicts an example of the samples and orientation.

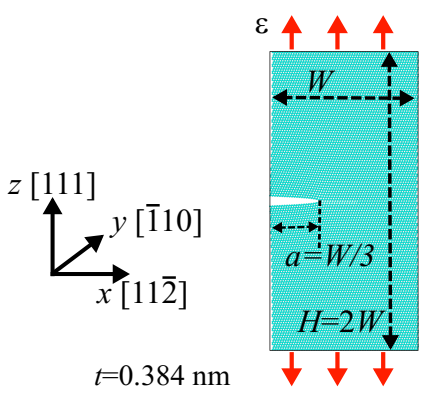

Fig. 1. Cracked samples employed in the molecular statistics analyses and orientation [23]. The thickness of the simulation cell is indicated as $t$.

Several specimens where considered, scaling their size until few nanometers: the width of the sample $W$ varied from $198 \mathrm{~nm}$ to $9.8 \mathrm{~nm}$, while the crack length $a$ was kept equal to $W / 3$. The mechanical properties were given by the following material constants: $\mathrm{C}_{11}=201 \mathrm{GPa}, \mathrm{C}_{12}=51.4 \mathrm{GPa}$ and $\mathrm{C}_{44}=90.5 \mathrm{GPa}$. Along the direction [111], an ideal material strength $\sigma_{\mathrm{IS}}$ of $35 \mathrm{GPa}$ at critical strain $\varepsilon \mathrm{C}=0.3$ was obtained. A stepwise increment of strain $\varepsilon$ is applied at the upper and lower layers of atoms according 
to Fig. 1, and the strain is increased until final fracture. At fracture, the critical displacement $d_{\mathrm{C}}$ (maximum displacement before failure) is obtained. The mechanical stress is obtained dividing the virial stress (stress $\times$ volume quantity) by the per-atom volume at $\varepsilon_{C}=0.3$, i.e. $24.44 \AA^{3}$. This value is, indeed, a more reliable estimation of the atomic volume in regions that are highly deformed, such as close to the crack tip at failure conditions. Analyses were conducted at $0 \mathrm{~K}$ and fully included the lattice trapping.

\section{$3 \quad$ Results and Discussion}

The crack tip stress fields of some selected considered geometries are presented in Fig. 2a for the sake of clarity. The values of the atoms at the crack tip are plotted at $r=0.1 \AA$ for convenience, since as well known the log-log scale does not allow the value of 0 . The depicted stress is the mechanical atomic stress, i.e. virial stress divided by the per-atom volume as explained in the previous section.

(a)

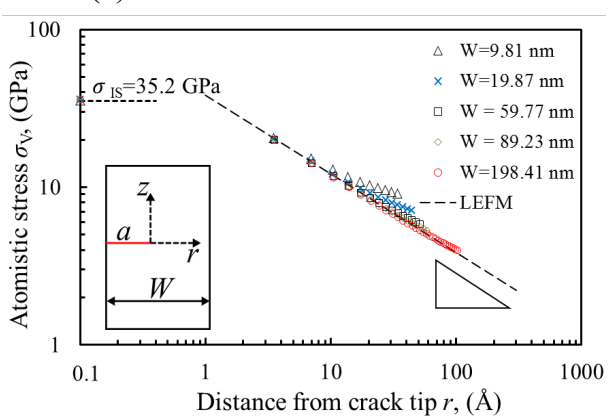

(b)

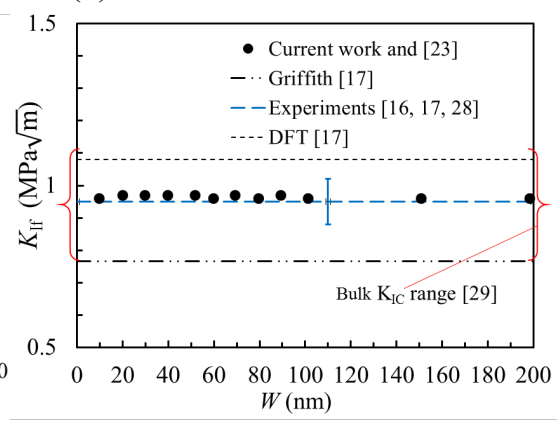

Fig. 2. (a) Near crack-tip atomic stress distribution for selected geometries; atomistic stress is derived from virial stress/per-atom volume. The stress values of the atoms at the crack tip are plotted for $r=0.1 \AA$ rather than $0 \AA$ in order to summarize in a single log-log scale graph both stress distribution and crack-tip values. (b) Critical stress intensity factors versus the variation of the specimen width $W$.

The figure shows two very important results:

- The MS analyses do not predict the infinite stress at the crack-tip but rather a finite value, i.e. the ideal material strength $\sigma$ Is, regardless of the specimen size. This indicates that the fracture, in the case of ideal brittle materials, is ultimately governed by atoms at crack tip.

- Even if the stress singularity is missing, near the crack tip region the stress still varies with $1 / r^{0.5}$ as expected from continuum LEFM. Fig. 2a depicts only selected geometries for the sake of clarity, but same results are obtained for all the considered samples. 
The results allow the quantification of the SIF at failure $K_{\text {If }}$ (i.e. at critical displacement $d_{\mathrm{C}}$ ), according to the classic Irwin's definition [26, 27]:

$$
K_{\mathrm{If}}=\sigma \mathrm{v} \sqrt{2 \pi r}
$$

where $\sigma_{\mathrm{v}}$ is the atomistic mechanical stress, perpendicular to the crack plane; $r$ is the distance from the crack-tip along the crack plane. The results are presented in Fig. $2 b$ and compared with other experimental and numerical works by other authors $[16,17$, 28 ]. $K_{\text {If }} \approx 0.97 \mathrm{MPa} \cdot \mathrm{m}^{0.5}$ is constant for all the geometries, and it agrees well with the fracture toughness of single crystal silicon, including bulk samples [29].

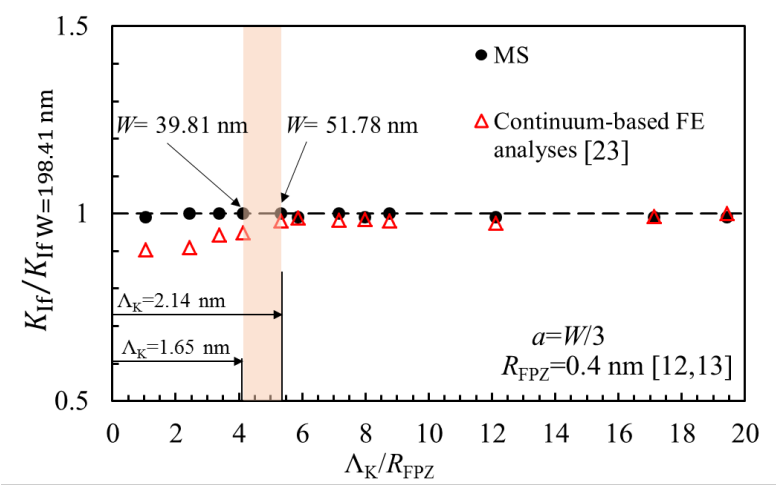

Fig. 3. Normalized SIF at failure versus the ratio of singular stress field length $\Lambda_{\mathrm{K}}$ and fracture process zone length $R_{\mathrm{FPZ}}$. Numerical values are reported in [23].

Finally, the comparison with continuum-based linear elastic solutions conducted in [23] is summarized in Fig. 3. The stress intensity factors at failure are normalized vs the $K_{\text {If }}$ of the largest sample $W=198.41 \mathrm{~nm}$, and plotted versus the length of the singular stress field $\Lambda_{\mathrm{K}}$ normalized by the fracture process zone $R_{\mathrm{FPZ}} . \Lambda_{\mathrm{K}}$ is the distance from the crack-tip at which the stress deviates more than $5 \%$ from the expected $1 / \mathrm{r}^{0.5}$, while the $R_{\mathrm{FPZ}}$ is a constant value taken from the literature $[12,13]$. When continuum and atomistic simulations are overlapped, the continuum-based formulation breaks down when the ratio between $\Lambda_{\mathrm{K}}$ and $R_{\mathrm{FPZ}}$ is approximately 4-5, in agreement with $[12,13]$. The atomistic simulations, instead, show a clearly scale-independence, confirming that ideal brittle fracture is ultimately governed by atomic bond breaking [7, 17].

Concluding, the concept of SIF is still surprisingly valid if the atoms are modeled, and static crack and ideal brittle material containing no other defects are considered. This result also agrees well with conclusions made by other authors [30]. Provided that the virial stress is affectively accepted as representative of atomic stress, crack tip region stress field of molecular system may be described by continuum-based SIF concept. 


\section{References}

1. Kitamura, T., Sumigawa, T., Shimada, T., Lich Van, L.: Challenge toward nanometer scale fracture mechanics. Eng Fract Mech 187, 33-44 (2018).

2. Kitamura, T., Sumigawa, T., Hirakata, H., Shimada, T.: Fracture Nanomechanics, 2 nd ed. Pan Stanford Publishing, Singapore (2016).

3. Xu, K., Li, B., Li, S., Luo, M., Gao, X., Jiang, C., Song, L.: In situ observation for the fatigue crack growth mechanism of 316L stainless steel fabricated by laser engineered net shaping. Int J Fatigue 130, 105272 (2019). doi: 10.1016/j.ijfatigue.2019.105272

4. Andric, P., Curtin, W.A.: Atomistic modeling of fracture. Model Simul Mater Sci Eng 27(1), 013001 (2018). doi:10.1088/1361-651x/aae40c

5. Ghayesh, M.H., Farajpour, A.: A review on the mechanics of functionally graded nanoscale and microscale structures. Int J Eng Sci 137, 8-36 (2019). doi:10.1016/j.ijengsci.2018.12.001

6. Taloni, A., Vodret, M., Costantini, G., Zapperi, S.: Size effects on the fracture of microscale and nanoscale materials. Nat Rev Mater 3, 211-224 (2018). doi:10.1038/s41578-018-0029-4

7. Shimada, T., Kitamura, T.: Fracture Mechanics at Atomic Scales. In: Altenbach H, Matsuda T, Okumura D (eds) Advanced Structured Materials. Springer International Publishing, Cham, pp. 379-396 (2015).

8. Kumar, S., Curtin, W.A.: Crack interaction with microstructure. Mater Today 10, 34-44 (2007) . doi:10.1016/S1369-7021(07)70207-9

9. Wang, Y.Q., Fritz, R., Kiener, D., Zhang, J.Y., Liu, G., Kolednik, O., Pippan, R., Sun, J.: Fracture behavior and deformation mechanisms in nanolaminated crystalline/amorphous micro-cantilevers. Acta Mater 180, 73-83 (2019). doi:10.1016/j.actamat.2019.09.002

10. Pippan, R., Wurster, S., Kiener, D.: Fracture mechanics of micro samples: Fundamental considerations. Mater Des 159, 252-267 (2019). doi:10.1016/j.matdes.2018.09.004

11. Gallo, P., Sumigawa, T., Shimada, T., Yan, Y., Kitamura, T.: Investigation into the Breakdown of Continuum Fracture Mechanics at the Nanoscale: Synthesis of Recent Results on Silicon. In: Gdoutos EE (ed) Proceedings of the First International Conference on Theoretical, Applied and Experimental Mechanics. Springer International Publishing, Cham, pp 205-210 (2019).

12. Gallo, P., Hagiwara, Y., Shimada, T., Kitamura, T.: Strain energy density approach for brittle fracture from nano to macroscale and breakdown of continuum theory. Theor Appl Fract Mech 103, 102300 (2019). doi:10.1016/j.tafmec.2019.102300

13. Shimada, T., Ouchi, K., Chihara, Y., Kitamura, T.: Breakdown of Continuum Fracture Mechanics at the Nanoscale. Sci Rep 5, 8596 (2015). doi:10.1038/srep08596

14. Gallo, P., Sumigawa, T., Kitamura, T., Berto, F.: Static assessment of nanoscale notched silicon beams using the averaged strain energy density method. Theor Appl Fract Mech 95, 261-269 (2018). doi:10.1016/j.tafmec.2018.03.007

15. Gallo, P., Sumigawa, T., Kitamura, T.: Experimental characterization at nanoscale of single crystal silicon fracture toughness. Frat ed Integrità Strutt 13, 408-415 (2019). doi:10.3221/IGF-ESIS.47.31

16. Gallo, P., Yan, Y., Sumigawa, T., Kitamura, T.: Fracture Behavior of Nanoscale Notched Silicon Beams Investigated by the Theory of Critical Distances. Adv Theory Simulations 1, 1700006 (2018). doi:10.1002/adts.201700006

17. Sumigawa, T., Shimada, T., Tanaka, S., Unno, H., Ozaki, N., Ashida, S., Kitamura, T.: Griffith Criterion for Nanoscale Stress Singularity in Brittle Silicon. ACS Nano 11, 62716276 (2017). doi:10.1021/acsnano.7b02493 
18. Huang, K., Shimada, T., Ozaki, N., Hagiwara, Y., Sumigawa, T., Guo, L., Kitamura, T.: A unified and universal Griffith-based criterion for brittle fracture. Int J Solids Struct 128, 67-72 (2017). doi:10.1016/j.ijsolstr.2017.08.018

19. Zhou, M.: A new look at the atomic level virial stress: On continuum-molecular system equivalence. Proc R Soc A Math Phys Eng Sci 459, 2347-2392 (2003). doi:10.1098/rspa.2003.1127

20. Cheng, S.H., Sun, C.T.: Convergence of local atomistic stress based on periodic lattice. Int J Solids Struct 51, 2027-2035 (2014). doi:10.1016/j.ijsolstr.2014.02.023

21. Subramaniyan, A.K., Sun C.T.: Continuum interpretation of virial stress in molecular simulations. Int J Solids Struct 45, 4340-4346 (2008). doi:10.1016/j.ijsolstr.2008.03.016

22. Zimmerman, J.A., Webblll, E.B., Hoyt, J.J., Jones, R.E., Klein, P.A., Bammann, D.J.: Calculation of stress in atomistic simulation. Model Simul Mater Sci Eng 12(4), S319S332 (2004). doi:10.1088/0965-0393/12/4/S03

23. Gallo, P.: On the Crack-Tip Region Stress Field in Molecular Systems: The Case of Ideal Brittle Fracture. Adv Theory Simulations 2, 1900146 (2019). doi:10.1002/adts.201900146

24. Plimpton, S.: Fast Parallel Algorithms for Short-Range Molecular Dynamics. J Comput Phys 117, 1-19 (1995). doi:10.1006/jcph.1995.1039

25. Sillinger, F.H., Weber, T.A.: Computer simulation of local order in condensed phases of silicon. Phys Rev B 31, 5262-5271 (1985). doi:10.1103/PhysRevB.31.5262

26. Irwin, G.R.: Linear fracure mechanics, fracture transition, and fracture control. Eng Fract Mech 1(2), 241-257 (1968).

27. Irwin, G.R.: Analysis of Stresses and Strains Near the End of a Crack Traversing a Plate. J. Appl. Mech. 24, 361-364 (1957).

28. Sumigawa, T., Ashida, S., Tanaka, S., Sanada, K., Kitamura, T.: Fracture toughness of silicon in nanometer-scale singular stress field. Eng Fract Mech 150, 161-167 (2015). doi:10.1016/j.engfracmech.2015.05.054

29. Stan, G., Krylyuk, S., Davydov, A.V., Cook, R.F.: Bending manipulation and measurements of fracture strength of silicon and oxidized silicon nanowires by atomic force microscopy. J Mater Res 27, 562-570 (2011). doi:10.1557/jmr.2011.354

30. Le, M.Q., Batra, R.C.: Mode-I stress intensity factor in single layer graphene sheets. Comput Mater Sci 118, 251-258 (2016). doi:10.1016/j.commatsci.2016.03.027 